\title{
Excision and Partial Primary Closure of Wound for Pilonidal Disease
}

\author{
Ahmed Lasheen*, Khalid Safwat, Mansour Morsy, Alaa Fiad, Abdelwahab Elmoregy \\ General Surgery Department, Faculty of Medicine, Zagazig University, Zagazig, Egypt \\ Email: *lasheenahmed@yahoo.com
}

Received April 15, 2012; revised May 21, 2012; accepted June 22, 2012

\begin{abstract}
Background: Pilonidal disease is a common chronic disorder of the sacrococcygeal region; and its surgical management is still a matter of discussion. The ideal therapy would be a quick cure that allowed patients to return rapidly to normal activity, that is minimally invasive with no morbidity, a low risk of complication and easy to learn. Methods: Sixty seven patients with pilonidal disease were subjected to excision and partial closure procedure, from November 2006 to March 2010 at General Surgery Department, Zagazig University Hospital, Zagazig University, Egypt. The mean age was 27.5 years. Complete but close excision of all diseased tissues was achieved. Then, the wound partially was closed from the deepest parts only by multiples buried vertical mattress sutures by using Lasheen's needle, and left the superficial wound parts to heal by second intention. The follow up period was ranged from $14-39$ months (mean 27 months). Results: The mean hospital stay was 12 hours, mean operation time was 30 minutes and mean time to return to normal activity was 8 days (range 7 - 12 days). Nine cases show superficial wound infection and one case of recurrence. Conclusion: Excision and partially closure technique by using Lasheen's needle for pilonidal diseases is minimal invasive and less morbidity, easy to learn and has favorable results regarding to return to normal activity, rate of recurrence and cosmetically acceptable.
\end{abstract}

Keywords: Partially Closure; Lasheen's Needle; Pilonidal Disease

\section{Introduction}

Pilonidal sinus is a disease that most commonly arises in the hair follicles of the natal cleft of the sacrococcygeal area. Incidence is reportedly 26 per 100,000 population, affecting males twice as often as females and predominantly young adults of working age [1]. Three factors should exist to initiate the pathology of the pilonidal sinus disease; the first is the presence of a well stimulated pilonidal dimple; the second factor is accumulation of hairs or cellular debris in the dimple; and the third factor is the power to introduce and charge the area to initiate the pathology. Thus, the midline scar alone without a nidus for initiate the pathology (suture, dimples and depression) is of no significant value as a precursor for recurrence [2]. The ideal therapy would be a quick cure that allows patients to return rapidly to normal activity, that is minimally invasive with no morbidity, and a low risk of complications [3]. The management of chronic pilonidal disease is variable, contentious, and problematic. Principle of treatment require eradication of the sinus tract, complete healing of the overlying skin, and prevention of recurrence [4]. The surgical wound may be

"Corresponding author. left to heal by open healing (secondary intention). Advocates of this technique state that reduced wound tension facilitates trouble free healing without recurrence if all sinus tracts are fully excised [5]. Alternatively, the wound may be closed to heal by primary closure, either by midline closure techniques (with the wound lying within the natal cleft) [6] or other techniques (where the wound is placed out with the midline) [7]. In this study, we used excision and partially closure as a novel minimally invasive approach for management of pilonidal disease.

\section{Patients and Methods}

Sixty seven patients with pilonidal disease (37 patients have pilonidal abscess and 30 patients have chronic pilonidal sinus) were subjected of this study. There were 57 men and 10 women with the mean age of 27.5 years (range from 18 to 31 years). Informed consent was obtained from all patients after the nature of the procedure was explained. Lasheen's needle was prepared by corresponding author, it is curved needle, its length ranged from $10-15 \mathrm{~cm}$, has two sharp pointed ends, and long hole (about one centimeter in length) at the middle of needle through it the thread (Vicryl No. 0) was passed Figure 1. The procedure was carried out under general 
anesthesia in 59 patients ( 37 pilonidal abscess cases and 22 chronic pilonidal cases) and local anesthesia in 8 patients (limited chronic pilonidal disease cases), with patient in a prone position with natal cleft was separated. After shaving off the hair and cleaning the area with povidine-iodine, complete but close excision of all pilonidal tissues was achieved. By using slowly absorbable suture No. 0 (braided coated glycolide homopolymer violet) was passed through middle hole of the needle. Multiples vertical buried mattress sutures were putted through the wound using Lasheen's needle to close the deepest parts of wound only. As follow, the Lasheen's needle with thread (Vicryl No. 0) passed in its hole, was passed through the wound edge midway between skin surface and floor of wound to came out through the skin about 2 $\mathrm{cm}$ of wound margin (only about two third of the needle appears outside the skin). At this point, the needle direction was changed, to pass by other needle end under the floor of the wound to appear from other wound side about $2 \mathrm{~cm}$ of wound margin (only about two third of needle appears outside the skin). Then, the needle direction was changed to pass first needle end, which still present inside in tissue through the wound edge to appear from the wound midway between skin surface and floor of wound. Then, the needle come out completely with thread (Vicryl No. 0) of tissue. After this last step, buried vertical mattress suture will be formed where, one limb was passed through one wound side midway between skin surface and floor of wound, then passed under the wound floor, and other suture limb passed through other wound side also midway between skin surface and floor of wound Figures 2(a)-(f) and Figures 3(a)-(b). The two strands of each suture were tied through the wound to close the deepest portion of wound (primary intention repair) and was make superficial portion near to each other (secondary intention repair). Light Gauze pack was put on wound edges and coved by dressing. Patients were encouraged to mobilize soon after the procedure. Oral broad spectrum antibiotic was prescribed for one week. Daily dressing of the wound was done by patient at home. The patients were observed for wound complications and recurrence for 14 - 39 months (mean 27 months).

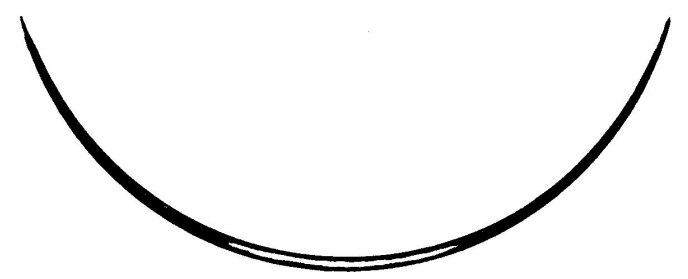

Figure 1. The Lasheen's needle. Curved needle, its length ranged from 10 to $15 \mathrm{~cm}$, has two sharp pointed ends and long hole (about one centimeter) at the middle of its length, and through it the thread (Vicryl No. 0 ) was passed.

\section{Results}

Pilonidal abscess symptoms and signs were the presentation in 37 patients and chronic pilonidal sinus symptoms and signs were presentation in 30 patients. The time of operation ranged from 20 to 45 minutes (mean, 30 minutes). The mean hospital stay was 12 hours (range, 8 - 18 hours). The mean time to return to normal activity was 8 days (range, 7 - 12 days). The average healing time was 8 days (range, 7 - 14 days). Nine cases (13.4\%) had early superficial wound infection ( 7 cases of pilonidal abscess cases and 2 cases of chronic pilonidal sinus cases), which was treated by twice daily dressing. Recurrence was occurred in one case $(1.4 \%)$ at 6 months postoperatively.

\section{Discussion}

Pilonidal disease predominantly affects younger populations and therefore this disease has an economic impact. Wounds heal more quickly after primary closure and return to work is sooner than with open healing, but at the expense of an increased risk of sinus recurrence. It would seem that treatment type should still be based on surgeon and patient preference, with consideration of patient's goals for therapy [4]. The management of the pilonidal abscess is drainage and removal of all necrotic tissues and this wound is left to heal by secondary intention [5]. Some surgeons prefer to close this wound after abscess excision primary [8]. The chronic pilonidal sinus management is controversial and opinions very about the ideal technique. Broadly, there are three types of management. Firstly, excision and healing by secondary intention, the principal advantages of this technique are easy, less invasive, low recurrence rate but this technique undoubtedly has high direct and indirect costs and the downside is a lengthy healing time (three to eight weeks). Despite this, there is a role for wide excision in those with extensive chronic disease and those operated for failed primary closure [9]. Secondly, excision and primary closure is more cosmetically acceptable for some patients and is associated with a shorter healing time and time off work. However, this potential benefit is offset by the need for bed rest for up to one week in hospital coupled with a high risk of postoperative infection [10]. The scar can be sited over the midline or displaced laterally with one year recurrence rates of $18 \%$ and $10 \%$ respectively [11]. Thirdly, excision with reconstructive procedures are more technically demanding and cosmetically not accepted for some patients. The aim for the majority of procedures is to reshape and flatten the natal cleft to reduce friction, local warmth and moisture, and hair accumulation $[7,12]$. In our procedure, after complete but close excision of all pilonidal diseased tissue, the deepest parts of wound are closed and the superficial parts of wound only are approximated by these buried sutures. So, 


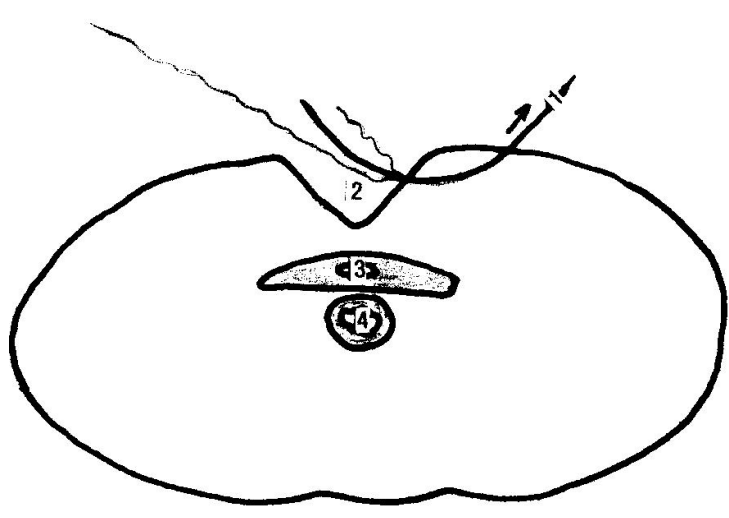

(a)

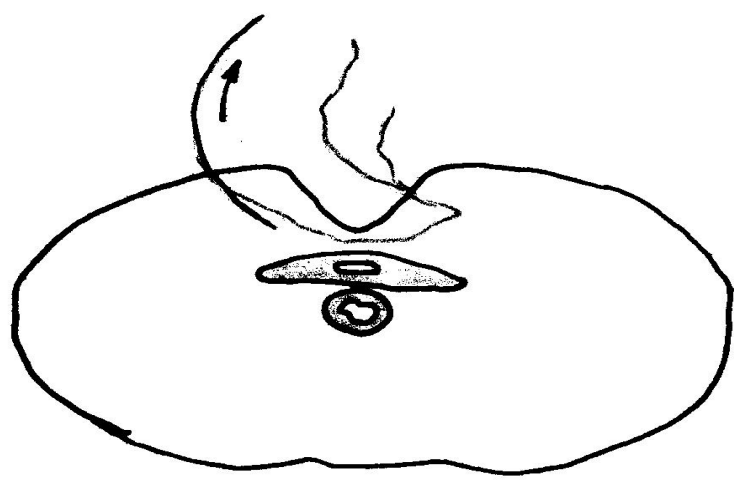

(c)

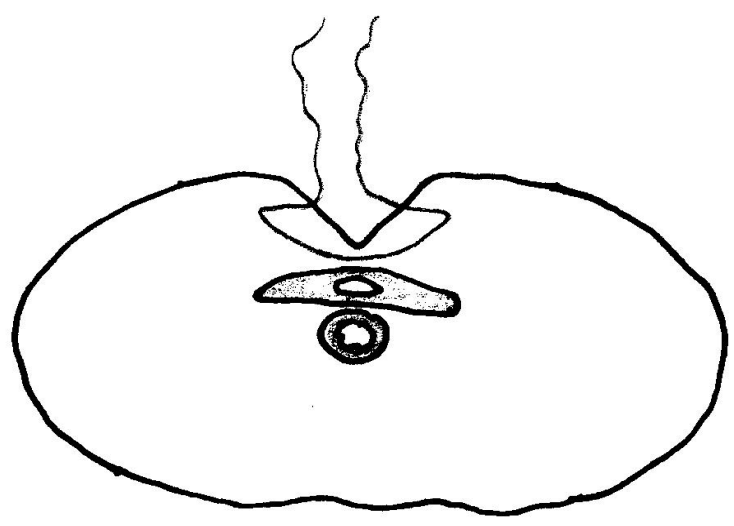

(e)

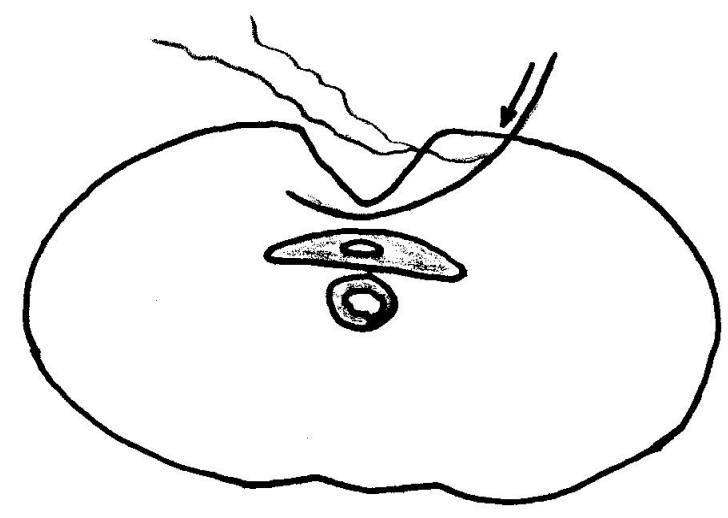

(b)

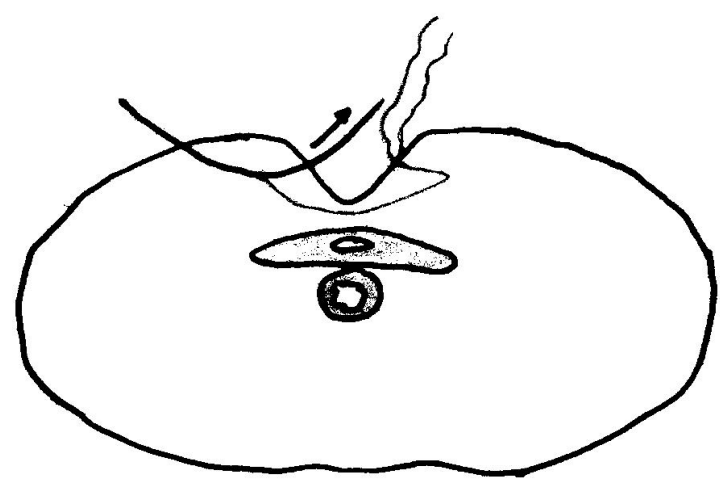

(d)

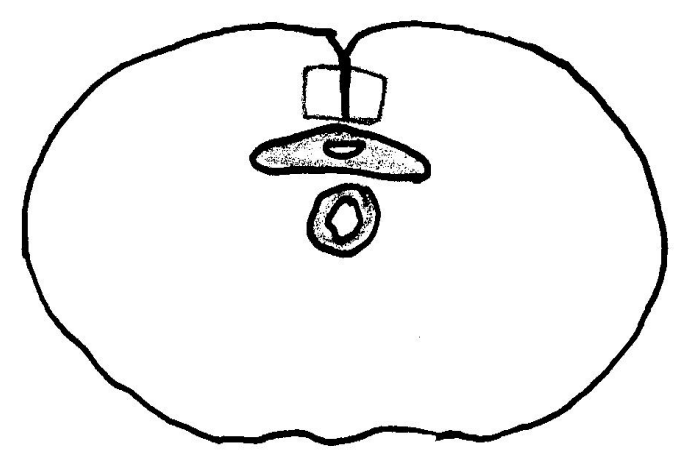

(f)

Figure 2. Diagram of excision and partially closure of pilonidal disease. Transverse cut section of patient in prone position at the plane of one vertical buried mattress suture and though the natal cleft wound. 1: Lasheen's needle and Vicryl No. 0 thread passed through its hole; 2: Natal cleft wound after excision of all pilonidal diseased tissue; 3: Sacrum; 4: Rectum. (a) Limited and good excision of all pilonidal diseased tissue was done. Lasheen's needle with slowly absorbable No. 0 (braided coated glycolide homopolymer violet) was passed through the wound edge midway between skin surface and floor of the wound to come out the skin about $2 \mathrm{~cm}$ of wound margin (only about two third of needle appears outside the skin); (b) The Lasheen's needle with slowly absorbable No. 0 thread was return after changed the direction of other needle end to pass under the floor of the wound and in its way to appear on other wound side (about $2 \mathrm{~cm}$ of wound margin); (c) Two third of Lasheen's needle came out from the skin about $2 \mathrm{~cm}$ from wound margin; (d) Then, Lasheen's needle end (first one) which still present in tissue was changed its direction to come out from the wound side midway between skin surface and floor of the wound. Complete withdrawal of needle to complete putting of suture was done; (e) After complete removal of Lasheen's needle, buried vertical mattress will be formed. Where, one limb was passed through one wound side midway between skin surface and wound floor, passed under the wound floor, and other limb suture passed through the other wound side, also between the skin surface and wound floor; (f) The two strands of each suture were tied through the wound to close the deep portion of wound to heal by primary intention and putting the superficial portion of wound near from each other to healed by secondary intention. 


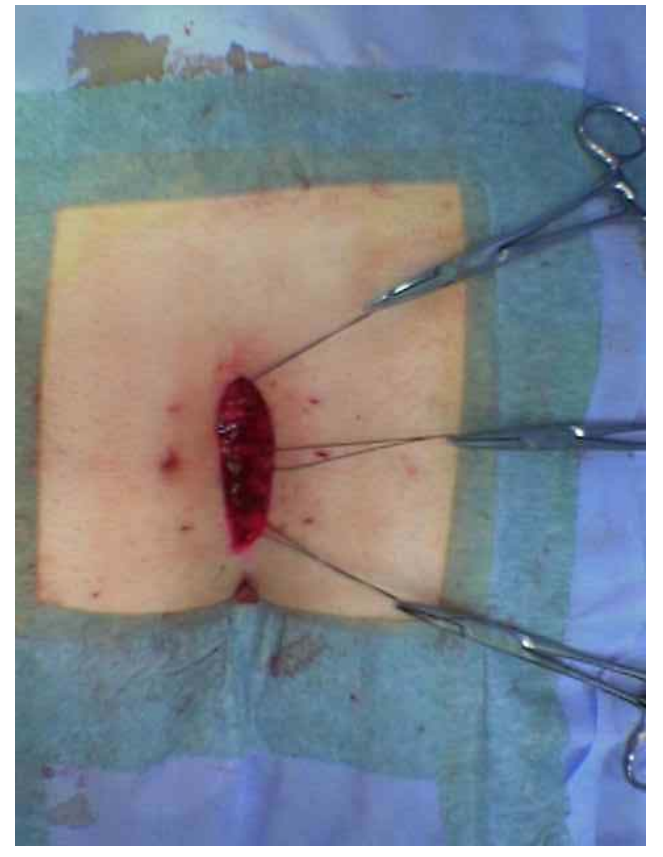

(a)

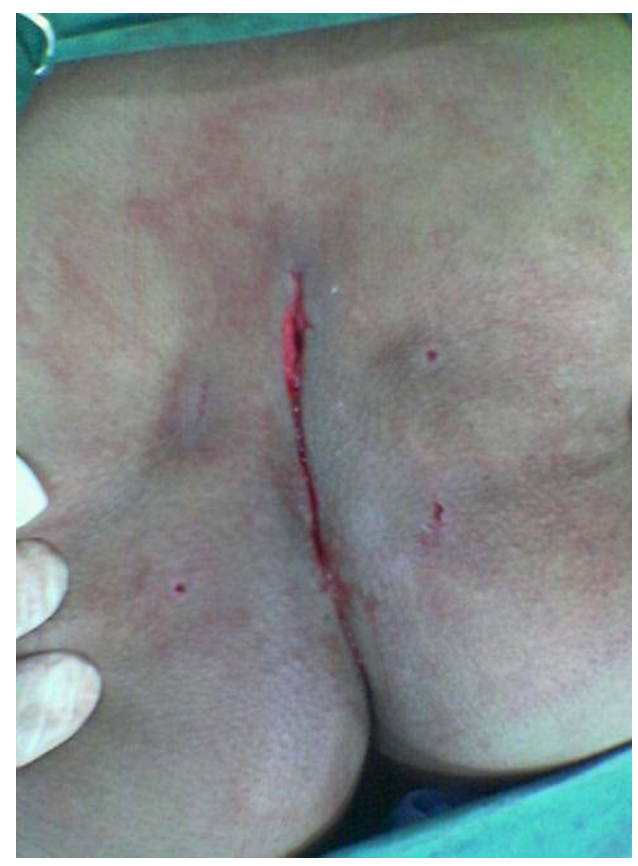

(b)

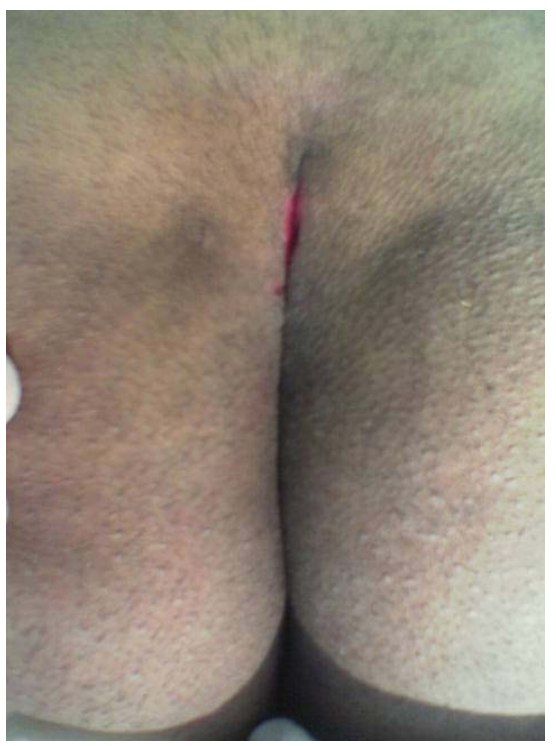

(c)

Figure 3. Excision with partial closure for pilonidal disease. (a) Limited and good excision of all diseased tissue at natal cleft was achieved and three buried mattress vertical sutures were put through the wound using Lasheen's needle. Each buried suture has one limb was passed through one wound side midway between skin surface and wound floor, passed under the wound floor, and other limb suture passed through the other wound side, also between the skin surface and wound floor; (b) The two strands of each suture were tied through the wound. When all sutures were tied, the deepest part of natal cleft wound will closed, which healed by primary intention and superficial part of wound becomes much reduced, which healed by secondary intention; (c) Same case after one week postoperatively, the wound was showed complete healing except small superficial part at the middle of the wound.

the deep parts of wound healed by primary closure and superficial parts of the wound healed by secondary intention after approximation of wound edges by these buried stitches.

Conclusion: Excision and partial primary closure pro- cedure is novel technique characterized by simple, easy to learn, less invasive and morbidity, reducing hospital stay, allowing early resumption of the work by patient and low recurrence rate. Also, this procedure is suitable for acute and chronic pilonidal disease. 


\section{REFERENCES}

[1] K. Søndenaa, E. Andersen, I. Nesvik and J. A. Søreide, "Patient Characteristics and Symptoms in Chronic Pilonidal Sinus Disease," International Journal of Colorectal Disease, Vol. 10, No. 1, 1995, pp. 39-42. doi:10.1007/BF00337585

[2] M. M. Awad, A. A. Elbaset, S. Ebraheem, E. Tantawy, M. A. Elhafez and A. M. Elsayed, "A Scoring System as a Method to Evaluate Pilonidal Sinus Disease to Make an Easy Decision for Its Management," Indian Journal of Plastic Surgery, Vol. 42, No. 1, 2009, pp. 43-48. doi:10.4103/0970-0358.53011

[3] T. L. Hull and J. Wu, "Pilonidal Disease," Surgical Clinics of North America, Vol. 82, No. 6, 2002, pp. 1169-1185. doi:10.1016/S0039-6109(02)00062-2

[4] J. McCallum, P. M. King and J. Bruce, "Healing by Primary Closure versus Open Healing after Surgery for pilonidal Sinus: Systematic Review and Meta-Analysis," British Medical Journal, Vol. 336, No. 7649, 2008, pp. 868871. doi:10.1136/bmj.39517.808160.BE

[5] M. Miocinović, M. Horzić and D. Bunoza, "The Treatment of Pilonidal Disease of the Sacrococcygeal Region by the Method of Limited Excision and Open Wound Healing," Acta Medica Croatica, Vol. 54, No. 1, 2000, pp. 27-31.

[6] I. Aldean, P. J. Shankar, J. Mathew, N. Safarani and N. Y. Haboubi, "Simple Excision and Primary Closure of Pilo- nidal Sinus: A Simple Modification of Conventional Technique with Excellent Results," Colorectal Disease, Vol. 7, No. 1, 2005, pp. 81-85. doi:10.1111/j.1463-1318.2004.00736.x

[7] E. Lasheen, K. Saad and M. Raslan, "Crossed Triangular Flaps Technique for Surgical Treatment of Chronic Pilonidal Sinus Disease," Archives of Surgery, Vol. 143, No. 5, 2008, pp. 503-505.doi:10.1001/archsurg.143.5.503

[8] Ommer, C. Pitt, K. Albrecht, B. Marla, K. Peitgen and M. K. Walz, "Pilonidal Sinus-Primary Closure also in Case of Abscess?" Zentralblatt für Chirurgie, Vol. 129, No. 3, 2004, pp. 216-219. doi:10.1055/s-2004-822742

[9] Kronborg, K. Christensen and C. Zimmermann-Nielsen, "Chronic Pilonidal Disease: A Randomized Trial with a Complete 3-Year Follow-Up," British Journal of Surgery, Vol. 72, No. 4, 1985, pp. 303-304. doi:10.1002/bjs. 1800720418

[10] D. J. Jones, "ABC of Colorectal Diseases. Pilonidal Sinus," British Medical Journal, Vol. 305, No. 6850, 1992, pp. 410-402. doi:10.1136/bmj.305.6850.410

[11] D. P. Berry, "Pilonidal Sinus Disease," Journal of Wound Care, Vol. 1, No. 2, 1992, pp. 29-32.

[12] R. Eryilmaz, M. Sahin, O. Alimoglu and F. Dasiran, "Surgical Treatment of Sacrococcygeal Pilonidal Sinus with the Limberg Transposition Flap," Surgery, Vol. 134, No. 5, 2003, pp. 745-749. doi:10.1016/S0039-6060(03)00163-6 